\title{
OPTIMAL DESIGN AND PLACEMENT OF PILOT SYMBOLS FOR CHANNEL ESTIMATION
}

\author{
Min Dong and Lang Tong \\ School of Electrical Engineering, Cornell University, Ithaca, NY 14853 \\ \{mdong,ltong\}@ee.cornell.edu
}

\begin{abstract}
The problem of design and placing pilot symbols for the estimation of frequency selective random channels is considered. For both SISO and MIMO channels, the Cramér-Rao Bound (CRB) on the mean square error of channel estimators is derived and minimized with respect to the pilot symbols and their placement. It is shown that placing pilot symbols, possibly in multiple clusters, in the middle of the data packet leads to minimum CRB.
\end{abstract}

\section{INTRODUCTION}

Channel estimation plays a critical role in packet-switched wireless systems where it is often necessary to acquire channel state for each packet. To facilitate channel estimation and synchronization, pilot symbols are usually used. For channels with short coherence time, the design of pilot sequence and its placement can affect significantly the overall performance of the wireless system $[2,3,4]$.

In this paper, we consider the optimal design of pilot symbols and their placement for channel estimation. Since different mobile users may choose different channel estimators, in searching for the optimal design of pilot sequence, it is desirable to use a criterion that is independent of specific estimators used by individual receivers. A natural choice is the $\mathrm{CRB}$ on the mean-square error (MSE), and the objective of the design of pilot sequence can be formulated as one that minimizes the CRB.

The main contributions of this paper are as follows. For both single-input and single-output (SISO) and multiple-input multipleoutput (MIMO) finite impulse response (FIR) random channels, we first obtain the expression of CRB as a function of pilot symbols and their placement. It is then shown that the CRB is minimized by placing pilot symbols that satisfy certain orthogonality conditions, possibly in multiple clusters, in the middle of the packet. While this confirms the advantage of using the midamble placement as in GSM, our results also suggest that some other placements are also optimal. One of such placements is the QPP- $\alpha$ (Quasi Periodic Placement) scheme that, under mild conditions, was shown to be optimal for decision feedback equalization (DFE) and also optimal in terms of maximizing channel capacity $[3,2,1]$.

The optimization of pilot symbols and their placement has not been investigated until recently, although the design of optimal pilot sequence for the conventional training based channel estima-

This work was supported in part by the Army Research Office under Grant ARO-DAAB19-00-1-0507 and the Multidisciplinary University Research Initiative (MURI) under the Office of Naval Research Contract N00014-00-1-0564. tors has been investigated by many [6]. In [7], Negi and Cioffi presented the optimal design for OFDM systems. Adireddy and Tong tackled this problem for DFE and for the purpose of maximizing channel capacity, assuming that the channel is known. Also related is [5] by de Carvalho and Slock who obtained expressions of CRB for deterministic channels and examined the placement of pilot symbols via computer simulations. In their case, no optimal strategy was found as the CRB for the deterministic channel model is also a function of the channel coefficient.

\section{PROBLEM STATEMENT}

\subsection{The Channel Model}

We first consider the estimation of an SISO linear channel with random impulse response $h_{k}$. Results for MIMO channels are presented in Sec 6. The system is described by

$$
y_{k}=\sum_{i=0}^{L} h_{i} s_{k-i}+n_{k},
$$

where $y_{k}$ is the received signal, $n_{k} \stackrel{\text { i.i.d. }}{\sim} \mathcal{C N}\left(0, \sigma_{n}^{2}\right)$ is an i.i.d. complex Gaussian noise, and the channel vector $\mathbf{h}=\left[h_{0}, \cdots, h_{L}\right]^{T}$ consists of zero mean i.i.d. random variables $h_{i} \stackrel{i . i . d .}{\sim} p(h)$. The input sequence $\left\{s_{k}\right\}$ is made of $\mathrm{P}$ pilot symbols $\left\{s_{p}[i]\right\}$ and $\mathrm{N}$ i.i.d. data symbols $\left\{s_{d}[i]\right\}$, where $s_{d}[i] \stackrel{i . i . d .}{\sim} p\left(s_{d}\right)$ with zero mean and variance $\sigma_{d}^{2}$. The power of the pilot symbols is defined as $\sigma_{p}^{2}=\frac{1}{P} \sum_{i=1}^{P}\left|s_{p}[i]\right|^{2}$. We further assume that the data, the channel and noise are jointly independent.

We use the vector channel model for the entire packet corresponding to $N$ data symbols and $P$ pilot symbols. Denoting $\mathbf{y}=\left[y_{N+P-L}, \cdots, y_{1}\right]^{T}, \mathbf{s}=\left[s_{1}, \cdots, s_{N+P}\right]^{T}$, we have the vector model

$$
\mathbf{y}=\mathcal{T}(\mathbf{h}) \mathbf{s}+\mathbf{n}=\mathcal{H}(\mathbf{s}) \mathbf{h}+\mathbf{n},
$$

where $\mathcal{T}(\mathbf{h})$ is a block Toeplitz matrix generated from $\mathbf{h}$ and $\mathcal{H}(\mathbf{s})$ a Hankel matrix generated from input $\mathbf{s}$

$$
\begin{aligned}
\mathcal{T}(\mathbf{h})= & \left(\begin{array}{ccccc}
h_{0} & \cdots & h_{L} & & \\
& \ddots & & \ddots & \\
& & h_{0} & \cdots & h_{L}
\end{array}\right)_{(N+P-L) \times(N+P)}, \\
\mathcal{H}(\mathbf{s})= & \left(\begin{array}{ccc}
s_{N+P} & \cdots & s_{N+P-L} \\
s_{N+P-1} & \nearrow & s_{N+P-L-1} \\
\vdots & \nearrow & \vdots \\
s_{L+1} & \cdots & s_{1}
\end{array}\right) .
\end{aligned}
$$




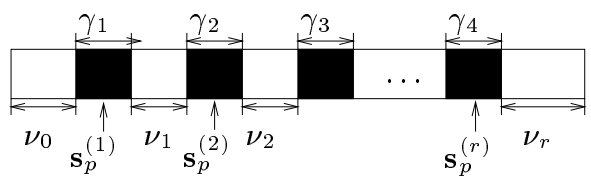

Fig. 1: A input sequence with multiple clusters

\subsection{Pilot Symbol Placement}

Consider the placement of $r$ clusters of pilot symbols as illustrated in Figure 1, which can be described by $\mathcal{P}=(\boldsymbol{\nu}, \boldsymbol{\gamma})$, where $\boldsymbol{\nu}=$ $\left[\nu_{0}, \cdots, \nu_{r}\right]$ is the cluster location vector and $\boldsymbol{\gamma}=\left[\gamma_{1}, \cdots, \gamma_{r}\right]$ the cluster length vector. For placements starting with pilot symbols, we have $\nu_{0}=1$, and those end with pilot symbols, $\nu_{r}=1$.

The data vector can be decomposed into the pilot and data parts

$$
\mathbf{s}=\mathbf{s}_{p}+\mathbf{s}_{d}
$$

where $\mathbf{s}_{p}$ is obtained by setting the data part of $\mathbf{s}$ to zero. This introduces a similar decomposition in the data matrix

$$
\mathcal{H}(\mathbf{s})=\mathcal{H}\left(\mathbf{s}_{p}\right)+\mathcal{H}\left(\mathbf{s}_{d}\right),
$$

and for convenience, we define their "autocorrelation" matrices as

$$
\mathbf{R}_{\mathbf{s}} \triangleq \mathcal{H}^{H}(\mathbf{s}) \mathcal{H}(\mathbf{s}), \quad \mathbf{R}_{\mathbf{s}_{p}} \triangleq \mathcal{H}^{H}\left(\mathbf{s}_{p}\right) \mathcal{H}\left(\mathbf{s}_{p}\right)
$$

Note that quantities $\mathbf{s}_{p}, \mathbf{s}_{d}$ and their corresponding correlation matrices are functions of placement $\mathcal{P}$.

For training based channel estimation, only those parts of the observation corresponding to the pilot symbols are used. Therefore, it is intuitive that all pilot symbols should be grouped into a single cluster. However, if all observations are used for channel estimation, i.e., the estimation is semiblind, it is not clear that pilot symbols should not be placed in multiple clusters. Indeed, the use of multiple clusters results in simpler design of pilot symbols as shown in Sec 4 and better detection performance [2].

\section{THE CRAMÉR-RAO BOUND}

The CRB is used as a performance measure for the design and placement of pilot symbols. The CRB on the minimum meansquare error in estimating a random parameter vector is well known $[8,9]$. The following theorem provides the expression of the CRB as a function of the pilot symbols and their placement.

Theorem 1 Let $\boldsymbol{\theta}=\left[\mathbf{s}_{d}^{H}, \mathbf{h}^{H}\right]^{H}$. Under the assumed model in Section 2, and regularity conditions [8, 9], the MSE of $\hat{\boldsymbol{\theta}}(\mathbf{y})$, defined as

satisfies

$$
\mathcal{M}(\hat{\boldsymbol{\theta}}) \triangleq E\left\{[\hat{\boldsymbol{\theta}}(\mathbf{y})-\boldsymbol{\theta}][\hat{\boldsymbol{\theta}}(\mathbf{y})-\boldsymbol{\theta}]^{H}\right\}
$$

$$
\mathcal{M}(\hat{\boldsymbol{\theta}}) \geq \mathbf{J}^{-1}\left(\mathcal{P}, \mathbf{s}_{p}\right)
$$

with the complex Fisher Information Matrix (FIM)

$$
\mathbf{J}\left(\mathcal{P}, \mathbf{s}_{p}\right)=\frac{1}{\sigma_{n}^{2}}\left(\begin{array}{cc}
E\left\{\mathbf{H}_{d}^{H} \mathbf{H}_{d}\right\}+\rho_{s_{d}}^{2} \mathbf{I} & \mathbf{0} \\
\mathbf{0} & E\left(\mathbf{R}_{\mathbf{s}}\right)+\rho_{h}^{2} \mathbf{I}
\end{array}\right)
$$

where $\mathbf{H}_{d}$ is obtained from $\mathcal{T}(\mathbf{h})$ by deleting columns corresponding to pilot symbols, $\rho_{s_{d}}^{2}=E\left\{\left[\frac{\partial \ln p\left(s_{d}\right)}{\partial s_{d}}\right]^{2}\right\}$ and $\rho_{h}^{2}=E\left\{\left[\frac{\partial \ln p(h)}{\partial h}\right]^{2}\right\}$. Treating $\mathbf{s}_{d}$ as the nuisance parameter vector, the complex CRBfor the MSE of channel estimators is given by

$$
\mathcal{M}(\hat{\mathbf{h}}) \geq \sigma_{n}^{2}\left(E\left(\mathbf{R}_{\mathbf{s}}\right)+\rho_{h}^{2} \mathbf{I}\right)^{-1} \triangleq \boldsymbol{\Lambda}\left(\mathcal{P}, \mathbf{s}_{p}\right) .
$$

From (9) we note that the FIM for $\boldsymbol{\theta}$ is block diagonal, and CRBs for the channel and data symbols are decoupled. Furthermore, the CRB for channel estimators is channel independent but is a function of pilot symbols $\mathbf{s}_{p}$ and placement $\mathcal{P}$, which makes the minimization of $\mathrm{CRB}$ with respect to $\mathbf{s}_{b}$ and $\mathcal{P}$ channel independent.

The optimal design of pilot symbols and their placement can now be formulated as finding $\mathcal{P}_{*}$ and $\mathbf{s}_{p_{*}}$ such that, for all $\mathcal{P}$ and $\mathbf{S}_{p}$ satisfying the power constraint,

$$
\operatorname{tr} \Lambda\left(\mathcal{P}_{*}, \mathbf{s}_{p_{*}}\right) \leq \operatorname{tr} \Lambda\left(\mathcal{P}, \mathbf{s}_{p}\right) .
$$

\section{OPTIMAL DESIGN FOR SISO CHANNELS}

We consider the design and placement of $r$ clusters of pilot symbols as shown in Figure 1. The placement of the clusters is specified by $\mathcal{P}=(\boldsymbol{\nu}, \boldsymbol{\gamma})$.

Theorem 2 Let $\lambda_{i}\left(\boldsymbol{\nu}, \boldsymbol{\gamma}, \mathbf{s}_{p}\right)$ be the ith diagonal entry of the CRB $\Lambda\left(\mathcal{P}, \mathbf{s}_{p}\right)$ with $\mathcal{P}=(\boldsymbol{\nu}, \boldsymbol{\gamma})$.

T2.1: For any $\mathbf{s}_{p}$ with power $\sigma_{p}^{2}$ and $\mathcal{P}=(\boldsymbol{\nu}, \boldsymbol{\gamma})$ such that $\nu_{0} \geq$ $L+1$ and $\nu_{r} \geq L+2$,

$$
\boldsymbol{\lambda}_{i}\left(\boldsymbol{\nu}, \boldsymbol{\gamma}, \mathbf{s}_{p}\right)=\boldsymbol{\lambda}_{i}\left(\boldsymbol{\nu}+\mathbf{e}_{1}, \boldsymbol{\gamma}, \mathbf{s}_{p}\right) \geq \frac{\sigma_{n}^{2}}{(N-L) \sigma_{d}^{2}+P \sigma_{p}^{2}+\rho_{h}^{2}}
$$

where $\mathbf{e}_{1}$ is the unit vector with 1 at the first entry and zero elsewhere, and the second equality holds iff $\mathbf{R}_{\mathbf{s}_{p}}=\sigma_{p}^{2} P \mathbf{I}$.

T2.2: Assume that pilot symbols satisfy, for $l=1, \cdots, L$,

$$
\begin{gathered}
\frac{1}{L-l+1} \sum_{i=1}^{L-l+1}\left|s_{p}[i]\right|^{2} \geq \sigma_{d}^{2}, \\
\frac{1}{L-l+1} \sum_{i=0}^{L-l}\left|s_{p}[P-i]\right|^{2} \geq \sigma_{d}^{2} .
\end{gathered}
$$

Then

$$
\min _{\boldsymbol{\nu}, \boldsymbol{\gamma}, \mathbf{s}_{p}} \lambda_{i}\left(\boldsymbol{\nu}, \boldsymbol{\gamma}, \mathbf{s}_{p}\right)=\frac{\sigma_{n}^{2}}{(N-L) \sigma_{d}^{2}+P \sigma_{p}^{2}+\rho_{h}^{2}},
$$

and the optimal placement is given by any $\boldsymbol{\nu}$ such that $\nu_{0}, \nu_{r} \geq L+1$ and $\mathbf{R}_{\mathbf{s}_{p}}=P \sigma_{p}^{2} \mathbf{I}$.

T2.1 indicates that for a certain groups of clusters, all the middle positions with fixed distances between clusters lead to the same CRB. If pilot symbols have sufficient power, T2.2 suggests that placing the clusters all in the middle leads to the lowest CRB. On the other hand, if the power of the pilot symbols is low, placing the pilot cluster at the two ends of the packet may have lower CRB. One such example is given in $\mathrm{Sec} 7$.

It can be shown that there always exists $\mathbf{s}_{p}$ such that the orthogonality condition in (2) holds. However, it is easier to find pilot symbols with this condition when multiple clusters are used. An interesting case is the placement using only a single pilot symbol in each cluster, i.e., $\gamma_{i}=1$. In such a case, pilot symbols placed $L+1$ apart and away from the two ends of the packet leads to the optimal design as shown in the following corollary.

Corollary 1 For any $\mathbf{s}_{p}$ with $\sigma_{p}^{2} \geq \sigma_{d}^{2}$ and any $\boldsymbol{\nu}^{*}$ such that $\nu_{i}^{*} \geq$ $L+1$,

$$
\begin{aligned}
\lambda_{i}\left(\boldsymbol{\nu}^{*}, \mathbf{1}, \mathbf{s}_{p}\right) & =\min _{\boldsymbol{\nu}, \boldsymbol{\gamma}, \mathbf{s}_{p}} \lambda_{i}\left(\boldsymbol{\nu}, \boldsymbol{\gamma}, \mathbf{s}_{p}\right) \\
& =\frac{\sigma_{n}^{2}}{(N-L) \sigma_{d}^{2}+P \sigma_{p}^{2}+\rho_{h}^{2}}
\end{aligned}
$$




\section{THE QUASI PERIODIC PLACEMENT}

The so-called Quasi Periodic Placement (QPP) [1] is a family of placement strategy that is shown to be optimal in the sense of maximizing mutual information when the channel is known. It is also optimal in the sense of minimizing average mean square error associated with transmitted symbol when a decision feedback equalizer is used, again, assuming known channel. Theorem 2 indicates that QPP is also optimal, in some sense, for channel estimation.

The QPP family is divided into different classes on the basis of the smallest allowable length of any pilot symbol cluster. The class of schemes for which $\alpha$ is the smallest allowable known symbol cluster length is denoted as QPP- $\alpha$.

Definition 1 Given an $\alpha$ and a frame with $N$ unknown symbols and $P$ known symbols, let $J=\left\lfloor\frac{P}{\alpha}\right\rfloor+1$. A placement scheme $\mathcal{P}(\boldsymbol{\mu}, \boldsymbol{\gamma})$ belongs to $Q P P-\alpha$ if and only if

$$
\begin{aligned}
& \text { 1. } \gamma_{i} \geq \alpha \text {, and } \sum \gamma_{i}=P ; \\
& \text { 2. } \nu_{i} \in\left\{\left\lfloor\frac{N}{J}\right\rfloor,\left(\left\lfloor\frac{N}{J}\right\rfloor+1\right)\right\} \text {, and } \sum \nu_{i}=N .
\end{aligned}
$$

In other words, in a QPP- $\alpha$ scheme, the known symbols are divided into as many clusters as possible under the constraint that each of them is no less than $\alpha$, and these clusters are placed such that the unknown symbol blocks are as "equal" as possible. From Theorem 2, it is apparent that any QPP- $\alpha$ scheme with $\nu_{0}, \nu_{r} \geq L+1$ and satisfies the orthogonality condition is optimal.

If the channel is known and a decision feedback equalizer (DFE) is implemented at the receiver, then the placement of pilot symbols that minimizes the average (symbol) MSE is given by a QPP with cluster size 1. It turns out that the constraint on pilot symbols for QPP-1 is the easiest to satisfy. In fact, any pilot symbols placed $L+1$ apart and away from the two ends of the packet leads to the optimal design as shown in the following corollary.

Corollary 2 If $\frac{N}{P} \geq L+1$ and $\sigma_{p}^{2} \geq \sigma_{d}^{2}$, then $Q P P-1$ is optimal.

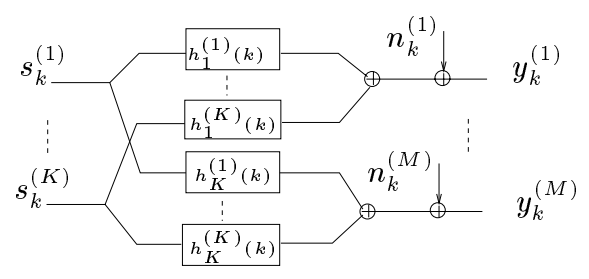

Fig. 2: A MIMO model

\section{OPTIMAL DESIGN FOR MIMO CHANNELS}

When there are more than one source, the system is modeled by a $K$-input $M$-output MIMO linear channel shown in Figure 2. Let $\mathbf{h}^{(i)}$ be the impulse response vector for the SIMO channel between the $i$ th user and the received $M$-dimensional vector $\mathbf{y}_{k}$ and $L_{i}$ the maximum channel order for the $i$ th user. The packet from user $i$ is denoted by $\mathbf{s}^{(i)}$ with data matrix $\mathcal{H}\left(\mathbf{s}^{(i)}\right)$. The MIMO channel can be described by

$$
\mathbf{y}=\mathcal{F}(\mathbf{s}) \mathbf{h}+\mathbf{n}
$$

where $\mathbf{s}$ and $\mathbf{h}$ are the stacking of the corresponding vectors $\mathbf{s}^{(i)}$, $\mathbf{h}^{(i)}$, and

$$
\mathcal{F}(\mathbf{s})=\left[\mathcal{H}\left(\mathbf{s}^{(1)}\right), \cdots, \mathcal{H}\left(\mathbf{s}^{(K)}\right)\right] \otimes \mathbf{I}_{M}
$$

is the overall input data matrix that includes both data and pilots.

Let $N_{i}, P_{i}$ be the number of data and pilot symbols for user $i$. Allowing pilots to be placed independently for each user, the placement $\mathcal{P}=(\boldsymbol{\nu}, \boldsymbol{\gamma})$ is defined by $\boldsymbol{\nu} \triangleq\left[\boldsymbol{\nu}^{(1)}, \cdots, \boldsymbol{\nu}^{(K)}\right], \boldsymbol{\gamma}=$ $\left[\boldsymbol{\gamma}^{(1)}, \cdots, \boldsymbol{\gamma}^{(K)}\right]$ where $\boldsymbol{\nu}^{(i)}$ and $\boldsymbol{\gamma}^{(i)}$ are the placement for user $i$. Given a placement $\mathcal{P}$, and the decomposition $\mathbf{s}=\mathbf{s}_{p}+\mathbf{s}_{d}$, the "autocorrelation" matrix associated with the pilot is defined by

$$
\mathbf{R}_{\mathbf{s}_{p}} \triangleq \mathcal{F}^{H}\left(\mathbf{s}_{p}\right) \mathcal{F}\left(\mathbf{s}_{p}\right)
$$

The CRB for channel estimators can be similarly derived as in SISO case with

$$
\mathcal{M}(\hat{\mathbf{h}}) \geq \sigma_{n}^{2}\left[\left(E\left(\mathbf{R}_{\mathbf{s}}\right)+\rho_{h}^{2} \mathbf{I}\right) \otimes \mathbf{I}\right]^{-1} \triangleq \mathbf{\Lambda}\left(\mathcal{P}, \mathbf{s}_{p}\right)
$$

The optimal pilot design and placement for MIMO channels can be summarized in the following theorem.

Theorem 3 Let $\lambda_{k}^{(i)}\left(\boldsymbol{\nu}, \boldsymbol{\gamma}, \mathbf{s}_{p}\right)$ be the CRB for the $k$ th channel coefficient of ith user.

T3.1: For any $\mathbf{s}_{p}$ with power $\sigma_{p}^{2}$ and $\mathcal{P}=(\boldsymbol{\nu}, \boldsymbol{\gamma})$ such that for all $i, \nu_{0}^{(i)} \geq L+1$ and $\nu_{r}^{(i)} \geq L+2$,

$$
\lambda_{i}\left(\boldsymbol{\nu}, \boldsymbol{\gamma}, \mathbf{s}_{p}\right)=\lambda_{i}\left(\overline{\boldsymbol{\nu}}, \boldsymbol{\gamma}, \mathbf{s}_{p}\right) \geq \frac{\sigma_{n}^{2}}{\left(N_{i}-L_{i}\right) \sigma_{d}^{2}+P \sigma_{p}^{2}+\rho_{h}^{2}}
$$

where $\overline{\boldsymbol{\nu}}$ is related to $\boldsymbol{\nu}$ by

$$
\bar{\nu}_{k}^{(i)}= \begin{cases}\nu_{k}^{(i)}+1 & k=1 \\ \nu_{k}^{(i)} & \text { otherwise }\end{cases}
$$

and the second equality holds iff $\mathbf{R}_{\mathbf{s}_{p}}=\sigma_{p}^{2} P \mathbf{I}$.

T3.2: Assume that $\left\{\mathbf{s}_{p}^{(i)}\right\}_{i=1}^{K}$ satisfies, for $l=1, \cdots, L_{i}$,

$$
\begin{gathered}
\frac{1}{L_{i}-l+1} \sum_{k=1}^{L_{i}-l+1}\left|\mathbf{s}_{p}^{(i)}(k)\right|^{2} \geq \sigma_{d}^{2}, \\
\frac{1}{L_{i}-l+1} \sum_{k=0}^{L_{i}-l}\left|\mathbf{s}_{p}^{(i)}(P-k)\right|^{2} \geq \sigma_{d}^{2} .
\end{gathered}
$$

Then

$$
\min _{\boldsymbol{\nu}, \boldsymbol{\gamma}, \mathbf{s}_{p}} \lambda_{k}^{(i)}\left(\boldsymbol{\nu}, \boldsymbol{\gamma}, \mathbf{s}_{p}\right)=\frac{\sigma_{n}^{2}}{\left(N_{i}-L_{i}\right) \sigma_{d}^{2}+P \sigma_{p}^{2}+\rho_{h}^{2}}
$$

and the optimal placement is given by any $\boldsymbol{\nu}$ such that

$$
\begin{aligned}
\nu^{(i)}(0), \nu^{(i)}\left(r_{i}\right) & \geq L_{i}+1, \quad \forall i \\
\mathbf{R}_{\mathbf{s}_{p}} & =\sigma_{p}^{2} P \mathbf{I} .
\end{aligned}
$$

Here $r_{i}$ is the number of clusters for user $i$.

Once more, the optimal strategy is to place pilot symbols in the middle of all data packets. The orthogonality condition in (26) for the pilot symbols, however, involves all users. The easiest way to satisfy this condition, perhaps, is the QPP-1 scheme extended for multiple users as suggested by the following corollary. 
Corollary 3 For any $\mathbf{s}_{p}$ with $\sigma_{p}^{2} \geq \sigma_{d}^{2}$ and any $\boldsymbol{\nu}^{*}$ such that

$$
\min _{i, j, k, l}\left|\sum_{m=0}^{k} \nu_{m}^{(i)}-\sum_{m=0}^{l} \nu_{m}^{(j)}\right| \geq \max _{i} L_{i}+1,
$$

we have

$$
\begin{aligned}
\lambda_{k}^{(i)}\left(\boldsymbol{\nu}^{*}, \mathbf{1}, \mathbf{s}_{p}\right) & =\min _{\boldsymbol{\nu}, \boldsymbol{\gamma}, \mathbf{s}_{p}} \lambda_{k}^{(i)}\left(\boldsymbol{\nu}, \boldsymbol{\gamma}, \mathbf{s}_{p}\right) \\
& =\frac{\sigma_{n}^{2}}{\left(N_{i}-L_{i}\right) \sigma_{d}^{2}+P \sigma_{p}^{2}+\rho_{h}^{2}} .
\end{aligned}
$$

The above result suggests that an optimal placement of pilot symbols, as illustrated in Figure 3, can be obtained if all pilot symbols are scattered in data packets in such a way that they are at least $L^{*} \triangleq \max _{i} L_{i}+1$ apart. The actual values of the pilot symbols are nonessential provided that they satisfy the power constraint.

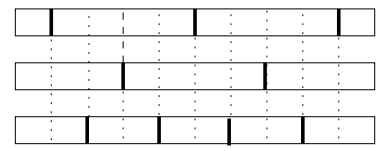

user 1

user 2

user 3

Fig. 3: A optimal placement for multiple users

\section{SIMULATION RESULTS}

We compare the performance of channel estimation for the multiuser case with different placement strategies through CRBs. Two tap channels were used with each tap i.i.d. Gaussian with zero mean and variance equal to 0.5 . Two users were considered with the same packet length that consisted of 52 data symbols and 15 pilot symbols. The QPP-(L+2) placement scheme was used in the simulation, the pilot symbols were grouped into 5 clusters with length 3 each and separated in such a way that the orthogonality condition of pilot symbols within the packet and between the users was satisfied, as similarly shown in Figure 3. We also considered each user with single cluster of pilot symbols and aligned at the same position for both users while the orthogonality condition was not satisfied. At last, we compared the performances of these two schemes with that of conventional training based estimation. Figure 4 left shows the trace of the CRB of the channel estimators in these three scenarios. Simulations indicate that some gain in the performance of channel estimation can be obtained by using optimal design and placement of pilot symbols. Moreover, as its easy design to satisfy the orthogonality condition, QPP- $\alpha$ scheme is attractive for optimal pilot symbols placement, particularly in the multiuser case. Finally, we gave an example of pilot symbols with low power case shown in Figure 4 right which plots the CRB vs. the position of a single cluster of pilot symbols. In this case, putting pilot symbols at the end of frame resulted in lower CRB.

\section{CONCLUSION}

We obtain the optimal design and placement of pilot symbols for the estimation of random channels by minimizing the CRB of channel coefficients. The optimal design and placement is derived in both SISO and MIMO channels. Our results show that for pilot symbols with sufficient power, placing them in the middle of the
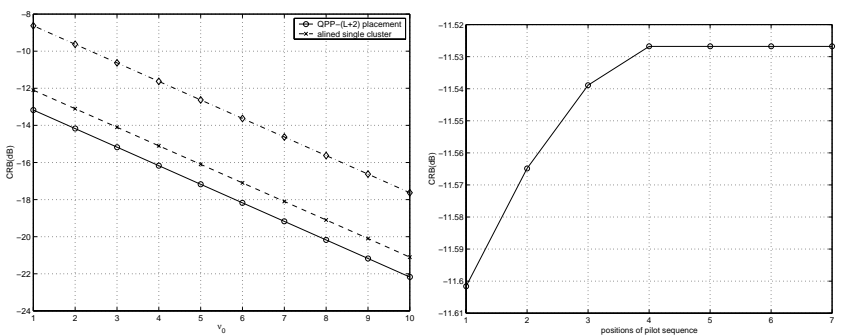

Fig. 4: Left: CRB of different placement schemes in multi-user case. Right: CRB vs. placements under low pilot power

data packet leads to minimum CRB. We also find that one of optimal placement is QPP- $\alpha$ scheme which is also optimal for DFE and maximizing channel capacity. Moreover, QPP-1 scheme leads to a easy design of optimal pilot symbols that satisfying the orthogonality condition.

For some transmission schemes where guard period is included before and after one packet, the placement of pilot symbols is no longer affect the CRB, especially in the single cluster case. The optimal designs of pilot symbols are those satisfy the orthogonality condition.

\section{REFERENCES}

[1] S. Adireddy and L. Tong. "Optimal Placement of Known Symbols". Submitted to IEEE Trans. Information Theory.

[2] S. Adireddy and L. Tong. "Detection with Embedded Known Symbols: Optimal Symbol Placement and Equalization”. In Proc. ICASSP'00 Conf., Istanbul, Turkey, June 2000.

[3] S. Adireddy and L. Tong. "Optimal Embedding of Known Symbols". In Proc. 34th Conf. Information Sciences and Systems, volume 1, March 2000.

[4] B.Hassibi and B.Hochwald. "How much Training is Needed in Multiple-Antenna Wireless Links". Submitted to IEEE Trans. Information Theory, August 2000.

[5] E. de Carvahlo and D. Slock. "Semi-blind Methods for FIR Multichannel Estimation". In Signal Processing Advances in Wireless and Mobile Communications, Giannakis, Hua, Stoica, Tong, Eds. Prentice Hall, pages 211-254, 2000.

[6] R. Heimiller. "Phase Shift Pulse Codes with Good Periodic Correlation Properties". IRE Transactions on Information Theory, pages 254-257, October 1961.

[7] R. Negi and J. Cioffi. "Pilot Tone Selection For Channel Estimation In A Mobile OFDM System ”. IEEE Trans. on Consumer Electronics, 44(3):1122-1128, Aug. 1998.

[8] H.L. Van Trees. Detection, Estimation and Modulation Theory, volume 1. Wiley, New York, 1968.

[9] E. Weinstein and A. Weiss. "A General Class of Lower Bounds in Parameter Estimation". IEEE Trans. Inform. Theory, 34(2):338-342, March 1988. 